\title{
Predicting 7-day, 30-day and 60-day all- cause unplanned readmission: a case study of a Sydney hospital
}

\author{
Yashar Maali', Oscar Perez-Concha ${ }^{1,2}$, Enrico Coiera ${ }^{1}$, David Roffe ${ }^{3}$, Richard O. Day ${ }^{4,5}$ and Blanca Gallego ${ }^{1 *}$
}

\begin{abstract}
Background: The identification of patients at high risk of unplanned readmission is an important component of discharge planning strategies aimed at preventing unwanted returns to hospital. The aim of this study was to investigate the factors associated with unplanned readmission in a Sydney hospital. We developed and compared validated readmission risk scores using routinely collected hospital data to predict 7-day, 30-day and 60-day allcause unplanned readmission.
\end{abstract}

Methods: A combination of gradient boosted tree algorithms for variable selection and logistic regression models was used to build and validate readmission risk scores using medical records from 62,235 live discharges from a metropolitan hospital in Sydney, Australia.

Results: The scores had good calibration and fair discriminative performance with c-statistic of 0.71 for 7 -day and for 30-day readmission, and 0.74 for 60-day. Previous history of healthcare utilization, urgency of the index admission, old age, comorbidities related to cancer, psychosis, and drug-abuse, abnormal pathology results at discharge, and being unmarried and a public patient were found to be important predictors in all models. Unplanned readmissions beyond 7 days were more strongly associated with longer hospital stays and older patients with higher number of comorbidities and higher use of acute care in the past year.

Conclusions: This study demonstrates similar predictors and performance to previous risk scores of 30-day unplanned readmission. Shorter-term readmissions may have different causal pathways than 30-day readmission, and may, therefore, require different screening tools and interventions. This study also re-iterates the need to include more informative data elements to ensure the appropriateness of these risk scores in clinical practice.

Keywords: Hospital readmission, Readmission risk scores

\section{Background}

Unplanned readmissions to hospital represent a significant burden to health care systems, patients and their families [1]. While not all readmissions can be prevented, there is a consensus that readmission rates across the world are too high and could be reduced through targeted interventions [2-6].

Estimates of how many readmissions are avoidable remain controversial. In the United States all-cause readmissions within 30 days from discharge in 2011 were

\footnotetext{
* Correspondence: blanca.gallegoluxan@mq.edu.au

${ }^{1}$ Centre for Health Informatics, Australian Institute of Health Innovation, Macquarie University, Level 6, 75 Talavera Rd, Sydney, NSW 2109, Australia Full list of author information is available at the end of the article
}

reported as $15 \%$, and $12 \%$ were estimated to be potentially preventable $[2,3]$. In the United Kingdom, the emergency 30-day readmission rate between 2004 and 2010 was 7\%, and the estimated rate of potentially preventable readmissions was $2 \%$ [6]. The Canadian Institute for Health Information reported a rate of 30-day unplanned readmissions of $8.5 \%$ [7]. The latest report on returns to acute care in New South Wales, Australia, estimated $16 \%$ returns within 30 days after hospitalization for common clinical conditions, and 10\% returns within 60 days after common elective surgical procedures [4]. Stroke patients were the most likely to return with a condition deemed to be potentially related to their initial stay, such as a complication or an adverse 
event ( $43 \%$ of returns). For elective knee replacement, the proportion of returns due to orthopaedic complications was $46 \%$ [4].

Many factors can contribute to unplanned readmissions [1, 3, 8-17]. Some are related to deficiencies in quality of care either during the index admission, in the community or in the transition of care. Morbidity and functional disability $[10,12]$, socioeconomic status $[3$, 13, 14], and discharge to long-term/nursing facilities [8] have been found to be important general risk factors. Preventable factors under the control of the hospital include management errors, surgical complications, medication related errors, and poor discharge procedures that do not properly involve patients, their relatives, general practitioners or aged-care workers $[15,18,19]$. Some local initiatives to support patients and their caregivers after discharge have been proven to help $[16,17]$ but wide adoption of sustainable interventions remains elusive. Given limited resources, it makes sense to target those readmissions that hospitals are best able to prevent and to tailor the costliest interventions to patients most likely to benefit from them. This strategy requires methods to accurately, and in a timely manner, estimate risk.

In order to identify the patients that could benefit from discharge planning strategies or other interventions aimed at preventing unwanted returns to hospital, several risk scores have been put forward. We have found six recent (from 2010) existing risk score models of all-cause, 30-day, unplanned [1], emergency, or potentially avoidable readmission: LACE index [20], LACE index + [21], Rothman index [22], HOSPITAL score [23], PARR-30 [24], and PREADM [25]. Typical c-statistic or area under the receiver operating characteristic curve (AUC) for these models ranges from 0.68 (LACE index [20] - prediction includes death -) to 0.75 (LACE index + [21] and Rothman index [22]). Their performance is only fair, when compared, for example, with predictions of mortality using similar data [26, 27]. Part of the problem may lie on the need for additional information, since potential predictors of unplanned readmission span beyond typically available clinical and administrative variables to include patient socio-economic information, patient living arrangements, hospital organisational factors, models of primary and community care available to patients, and patient preferences [28].

In this study, we explored readmission patterns and predictors for all-cause unplanned readmission within 7 days, 30 days and 60 days following discharge from a metropolitan hospital in Sydney, Australia. We utilized routinely available hospital Electronic Health Record (EHR) data together with administrative information on admissions to all other hospitals within the State, which are routinely collected by the Department of Health and linked to the hospital record. We first built a set of predictive models based on a gradient tree boosting algorithm [29]. In the presence of noisy correlated categorical data with unknown interactions, these types of machine learning methods are preferable to the more common logistic regression models [30]. Features selected by these predictive models were then used to develop simple scores, which can be readily used in a hospital setting. Risk scores have less accuracy than their corresponding gradient tree boosting methods but are easy to use in the clinical setting and easy to interpret by users. Patterns and predictors of 7-day versus longerterm readmission were compared.

\section{Methods}

\section{Settings and study population}

Electronic Health Records (EHRs) from 77,776 patients admitted to a 350-bed Sydney teaching, metropolitan hospital between 1 July 2008 and 31 December 2012 were collected. For each patient, an index admission was defined as the first hospital admission by the patient during the study period. Records in the one year before the index admission and 2 months after the index admission from all hospital admissions, emergency department visits and deaths within the State of New South Wales (NSW) were extracted from population health datasets. Namely, the NSW Admitted Patient Data Collection (APDC), the NSW Emergency Department Data Collection (EDDC), and the NSW Registry of Births, Deaths and Marriages (RBDM). The linkage amongst the APDC, EDDC and RBDM was performed by the NSW Centre for Record Linkage using a probabilistic linkage procedure, which guarantees false positive rates $<0.5 \%$ and false negative rates $<0.1 \%$ [31]. The linkage between the hospital EHR and the NSW administrative datasets was also carried out independently by the NSW Centre for Record Linkage and only 17 patients could not be linked to the APDC.

Of all 77,759 index admissions, 62,255 patients $(80.1 \%)$ were discharged alive by hospital, $15.2 \%$ were followed by transfers to hospitals, nursing homes or other facilities, $2.0 \%$ died during admission, $2.0 \%$ were discharged at own risk, and the reminder $0.7 \%$ represented discharges on leave, changes in type of care or had missing discharge information (Fig. 1).

\section{Definitions of readmission}

A readmission was defined as the first admission to any hospital in New South Wales (NSW) within 60 days of being discharged alive from the index admission. Subsequent readmissions by the same patient or readmissions beyond 60 days were ignored for the purpose of this study. A readmission was defined as 


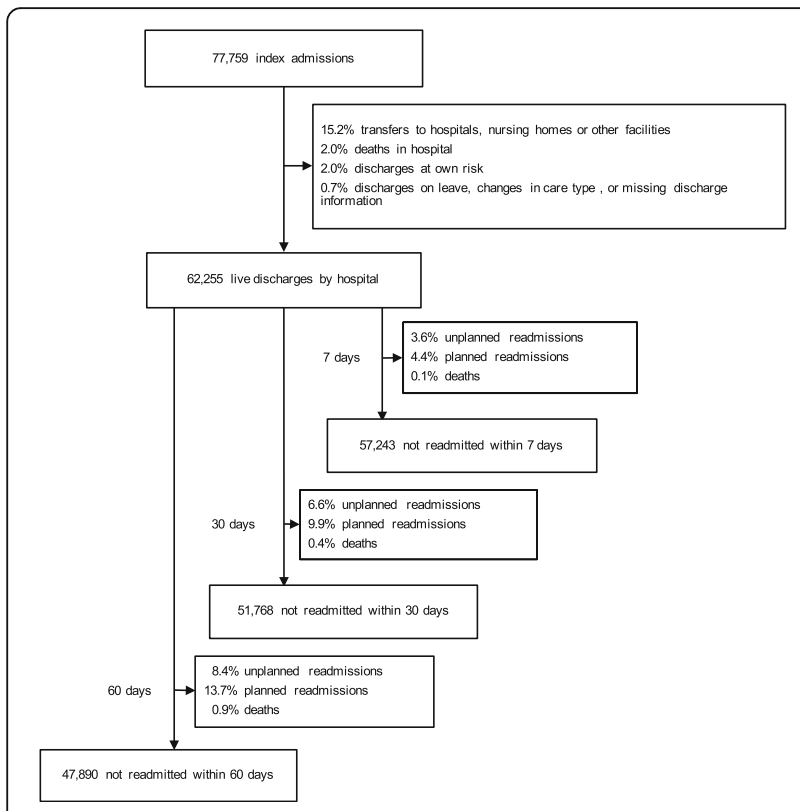

Fig. 1 Summary of patients discharged and readmitted over three overlapping periods: 7-days, 30-days and 60-days postdischarge

unplanned if it was initiated via the emergency department (ED). Amongst the 62,255 patients discharged alive, 13,818 (22.2\%) had a readmission within 60 days (5258 of those were unplanned readmissions; $8.4 \%$ of the total alive discharges) and 547 died during the follow up period (see Fig. 1). Readmissions were further stratified as occurring within 7 days, 30 days or 60 days from discharge from the index admission.

\section{Potential predictors}

Patients and admissions were characterized using 88 commonly-used variables available in the electronic health record, which can be divided into 5 categories:

1) Patient demographics: age, sex, marital status and payment status.

2) Patient acute-care history: information on cumulative length of stay (LOS) of hospital admissions within the previous year, as well as time since last admission.

3) Patient clinical status: Elixhauser comorbidity groups [32] (defined including one year hospital history), two last common pathology results available before discharge, including hours since last pathology panel. Pathology tests were grouped by their corresponding pathology panels. Results for each panel were classified as missing (if no pathology test within the panel was performed), abnormal (if any test result within the panel was abnormal), or normal.
4) Admission type: principal diagnosis, principal procedure type, duration of surgery, type of care, source of referral to hospital, arrival mode and triage code (if coming through ED), ward allocation, LOS, and number of pathology tests and surgeries performed.

5) Admission and discharge times: day of the week and time of the day of admission and discharge.

A detailed description of these variables and their distribution in the study population can be found in Additional file 1: Tables S2-S6.

\section{Pre-processing}

Continuous variables were first discretized into categorical variables taking into account domain knowledge and their distribution (Additional file 1: Table S2). Categorical variables were then separated into 211 independent binary variables. The data was separated randomly into two sets: a derivation set consisting of $80 \%$ of the records and used to derive the final scores and a validation set for evaluation.

\section{Gradient tree boosting models}

Separate models were built to predict readmissions within 7 days, 30 days and 60 days from discharge. Each predictive model was built and evaluated using 10 -fold cross validation on the derivation set. Patients with a planned readmission were ignored and removed from the derivation and validation datasets. A gradient tree boosting [29] algorithm was used for prediction. Gradient tree boosting is a machine learning technique that combines the prediction of an ensemble of weak regression trees, which are added sequentially to the model in order to maximize predictive performance and minimize model complexity. In this study we used the freely available gradient tree boosting algorithm implemented in the $\mathrm{R}$ package XGBoost [33] (see Additional file 1: Table S1 for description of model parameters).

\section{Feature selection}

Feature importance was initially quantified using the measure gain provided by XGBoost. Gain represents the improvement in regularized AUC obtained in each split. It is estimated for each feature of each tree and then averaged over all trees. In each cross-validation trial, the top 25 most important features found by the XGBoost algorithm (accounting for over 90\% of gain) were selected and included in a logistic regression model. A set of regression parameters was obtained averaging over all cross-validation trials. Statistically significant features ( $p$-value $<0.05)$ for more than $50 \%$ of the trials were retained. The mean of the selected features' distributions 
for index admissions followed by 7-day readmission, those followed by a readmission between 8 and 30 days and those followed by a readmission between 31 and 60 days were compared using t-test statistics. This comparison was carried out to explore the change of the distribution of important features over different time periods.

\section{Risk scores}

A set of readmission risk scores RETURN7, RETURN 30 and RETURN 60 were created using the averaged regression parameters of the selected variables. Following Donze et al. [23], scores for each selected variable were assigned by dividing regression parameters by the smallest one and rounding them to the nearest integer.

\section{Model performance}

The discriminative ability of the gradient tree boosting models and the corresponding logistic regression models was estimated via the c-statistic or AUC. We also calculated the sensitivity, specificity and positive predictive value (PPV). For each model, thresholds for these measures were chosen as those that optimized the sum of sensitivity and specificity in the training sets. Both, the average and the standard deviation of these performance measures across all cross-validation trials within the derivation set were reported. The performance of the risk scores was evaluated in the validation set also using AUC, sensitivity, specificity and PPV. Calibration in the validation set was measured via the Hosmer-Lemeshow goodness-of-fit statistics of the observed and expected rate of unplanned readmission across different bins.

\section{Results}

Amongst the 62,255 discharges by hospital, 5258 patients (8.4\%) returned to hospital via ED within 60 days from discharge, 4101 (6.6\%) within 30 days and 2241 (3.6\%) within 7 days. The left panel of Fig. 2 shows the rate of planned and unplanned readmissions per day up to 30 days post-discharge. As expected, most readmissions took place shortly after hospitalization. Planned readmissions (hospitalizations not initiated via ED), peaked at weekly intervals post-discharge, reflecting planned weekly returns to hospital. In contrast, unplanned readmissions showed an exponential decrease in the number of readmissions from day of discharge. The majority of unplanned readmissions (65.6\%) were assigned an urgent to very urgent ED triage category, requiring treatment within $30 \mathrm{~min}$ of presentation. A large percentage of readmissions (37\% of unplanned) took place in a different hospital from the index hospital (see right panel in Fig. 2).

The cross-validated predictive performance of the gradient tree boosting models and their corresponding logistic regression models in the derivation set are reported in Table 1. The models achieved a fair performance with AUC for the gradient tree boosting models of 0.71 (for 7-day readmission, which has a very imbalanced dataset where classification categories are very unequally represented), 0.74 (for 30-day readmission) and 0.76 (for 60 -day readmission). Table 1 also reports performance measures for the risk scores in the validation set. Risk score discriminative power remained fair, with a small loss of performance compared to the full model. AUC was 0.71 for RETURN7 and RETURN30 and 0.74 for RETURN60. Sensitivity, specificity and PPV for these scores are reported in Table 1 using the cut-off score that maximised the sum of the sensitivity and specificity in the training sets. Values at additional cut-off points are reported in the Additional file 1: Table S8.

Hosmer-Lemeshow statistics measuring the fit between observed and expected readmission rates showed good calibration for all scores. Observed and expected rates for selected scores can be found in Table 2 and Additional file 1: Table S7.

A list of the features used to calculate the scores and their odds ratio (OR) can be found in Table 3. History of hospital admission in the last year, and in particular,
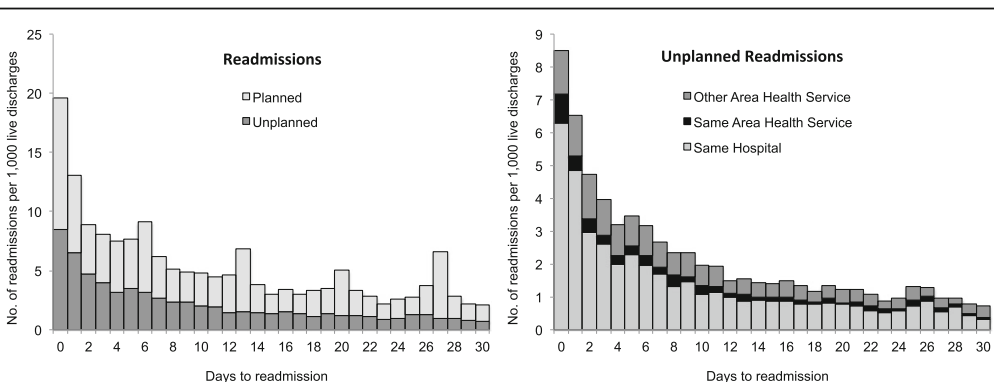

Fig. 2 Left panel: Number of planned and unplanned readmissions per 1000 live discharges, per day up to 30 days post-discharge. Right panel: Number of unplanned readmissions per 1000 live discharges, per day up to 30 days post-discharge to the same hospital, other hospitals within the same area health services (AHS) and other hospital in other AHS. Here readmission refers only to the first readmission after discharge. Subsequent readmissions by the same patient have been ignored 


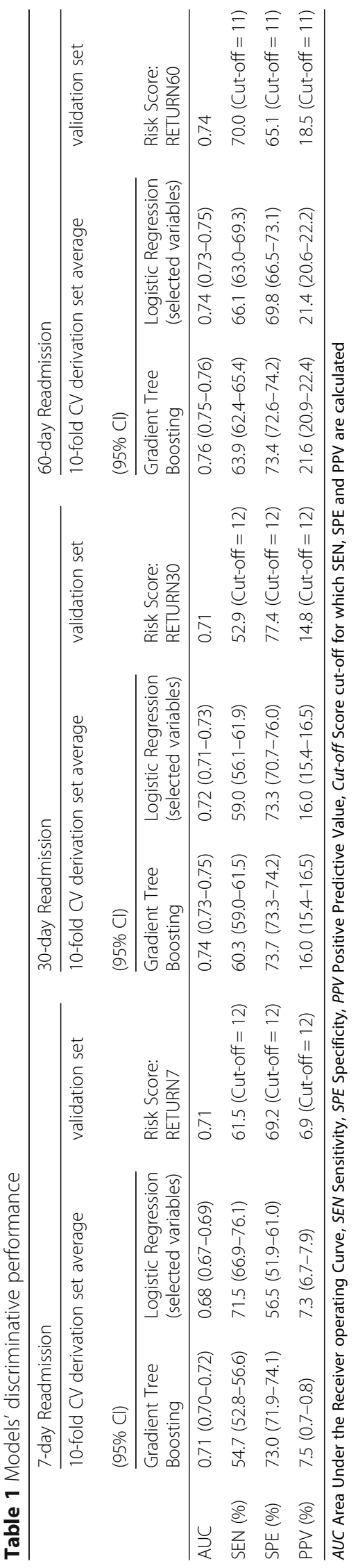


Table 2 Risk Scores' calibration performance

\begin{tabular}{|c|c|c|c|c|c|c|c|c|c|}
\hline & \multicolumn{3}{|c|}{ 7-day Readmission $\left(\beta_{0}=-4.72, \quad \beta_{1}=0.14\right)$} & \multicolumn{3}{|c|}{ 30-day Readmission $\left(\beta_{0}=-4.02, \quad \beta_{1}=0.13\right)$} & \multicolumn{3}{|c|}{ 60-day Readmission $\left(\beta_{0}=-3.79, \quad \beta_{1}=0.13\right)$} \\
\hline & $\begin{array}{l}\text { Number of } \\
\text { admissions }\end{array}$ & $\begin{array}{l}\text { Observed } \\
\text { readmission } \\
\text { rate }(\%)\end{array}$ & $\begin{array}{l}\text { Expected } \\
\text { readmission } \\
\text { rate }(\%)\end{array}$ & $\begin{array}{l}\text { Number of } \\
\text { admissions }\end{array}$ & $\begin{array}{l}\text { Observed } \\
\text { readmission } \\
\text { rate }(\%)\end{array}$ & $\begin{array}{l}\text { Expected } \\
\text { readmission } \\
\text { rate }(\%)\end{array}$ & $\begin{array}{l}\text { Number of } \\
\text { admissions }\end{array}$ & $\begin{array}{l}\text { Observed } \\
\text { readmission } \\
\text { rate }(\%)\end{array}$ & $\begin{array}{l}\text { Expected } \\
\text { readmission } \\
\text { rate }(\%)\end{array}$ \\
\hline score $=0$ & 641 & 0.6 & 0.9 & 185 & 1.1 & 1.8 & 135 & 2.2 & 2.2 \\
\hline score $=10$ & 1003 & 3.4 & 3.4 & 893 & 5.8 & 6.3 & 707 & 8.5 & 7.6 \\
\hline score $=20$ & 98 & 12.2 & 12.0 & 101 & 21.8 & 20.4 & 156 & 22.4 & 23.2 \\
\hline score $=30$ & 7 & 28.6 & 34.9 & 10 & 50.0 & 49.1 & 18 & 55.6 & 52.5 \\
\hline
\end{tabular}

Readmission Risk $(S)=\frac{e^{\left(\beta_{0}+S \cdot \beta_{1}\right)}}{1+e^{\left(\beta_{0}+5 * \beta_{1}\right)}}$, where $S=$ score, $\beta_{0}=$ intercept, $\beta_{1}=$ normalization parameter (full details in Additional file 1: Table S7)

cumulative $\mathrm{LOS}>7$ days (OR equal to $1.79,2.17$ and 3.52 for RETURN7, RETURN30 and RETURN60 respectively) and previous admission in the last 30 days (OR equal to 1.96, 2.18 and 1.53 for RETURN7, RETURN30 and RETURN60 respectively) were good predictors of future unplanned readmission in all risk scores. Old age and whether the index admission was an emergency admission was also important (see Table 3). Amongst the comorbidity groups, solid tumor without metastasis, psychosis and drug abuse were the prevalent predictors in all readmission groups. An abnormal test result before discharge as part of the frequently performed pathology panels: full blood count (FBC), Urea, Electrolytes and Creatinine (UEC), or Liver Function Tests (LFT), was also a predictor of readmission. In two situations (lipase for 7-day readmission and INR for 60-day readmission), in which most of the test results were either normal or missing, normal results versus no tests were associated with unplanned readmission. The two socio-economic variables (marital status and payment status) that were available in the medical record appeared important, as unmarried public patients were more likely to be readmitted. Overseas visitors had less probability of unplanned readmission at 60-days, probably reflecting lack of follow-up.

Some features, such as time since last surgery, last ward or discharge time, which were important for predicting 7-day readmission, were less important for predicting longer-term readmission. Conversely, variables such as LOS, cumulative LOS (cumLOS) in the past year, age over 85 and allied health intervention were better predictors of 30-day and 60-day readmission. Figure 3 shows the distributions of risk scores features across index admissions followed by 7-day unplanned readmission that were statistically different from the means features' distributions across index admissions followed by 8 to 30-day unplanned readmission. Unplanned readmission after the first week post-discharge was associated with longer index admission (25\% had LOS $>7$ days, compared to $17 \%$ for 7-day readmission). They were also associated with sicker patients with average number of comorbidity groups 2 (versus 1 for 7 -day readmission) and higher use of acute care in the past year. Differences in feature distributions between the 8-30 day and 31-60 day readmission groups were less significant.

\section{Discussion}

The aim of this study was to investigate the factors associated with unplanned readmission in a Sydney hospital. We started by measuring the number of unplanned readmissions per day to the same hospital, as well as to other hospitals within the State. We then developed and compared validated readmission risk scores using routinely collected hospital data to predict 7-day (RETURN7), 30-day (RETURN30) and 60-day (RETURN60) all-cause unplanned readmission. The AUC was 0.71 for RETURN7 and RETURN30 and 0.74 for RETURN60.

Given that hospitals struggle to keep up with growing demands from a rising number of hospitalizations, and that not all readmissions can be prevented, it is important to target interventions to patients that are most likely to benefit from them. Some strategies that have proved to be beneficial are costly and require additional qualified staff. As a result, they have not been widely adopted [34]. In this scenario, risk scores are an appropriate and easy-to-implement tool that can help identify high-risk patients before discharge. This has the potential to help target those for whom readmissions can be avoided for example with special transitional care, delayed discharge or provision of alternative care.

In this study, we found that a significant number of unplanned readmissions took place in hospitals different from the hospital of the index admission (see right panel of Fig. 2). This was confirmed in the latest report on readmissions in New South Wales [4]. It reflects the importance of maintaining medical record systems that are patient (as opposed to visit) centric, and can follow the patient across institutions [35]. It also has implications for the implementation of financial penalties for unplanned returns to hospital.

When compared to existing models of 30-day unplanned readmission, our model confirms much of what has already been observed in previous work [20-25]. Our predictive power is similar to that of the best 


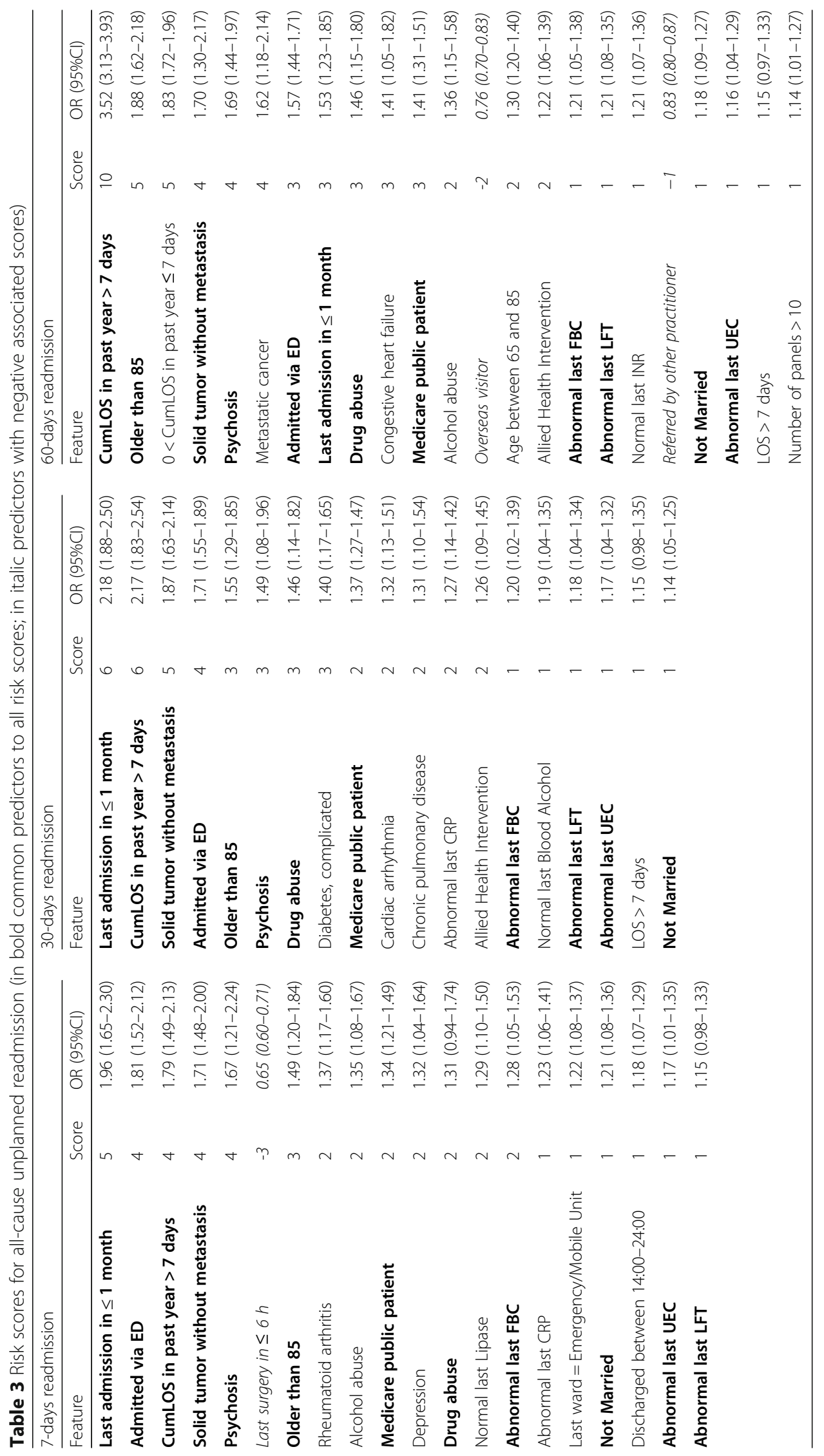




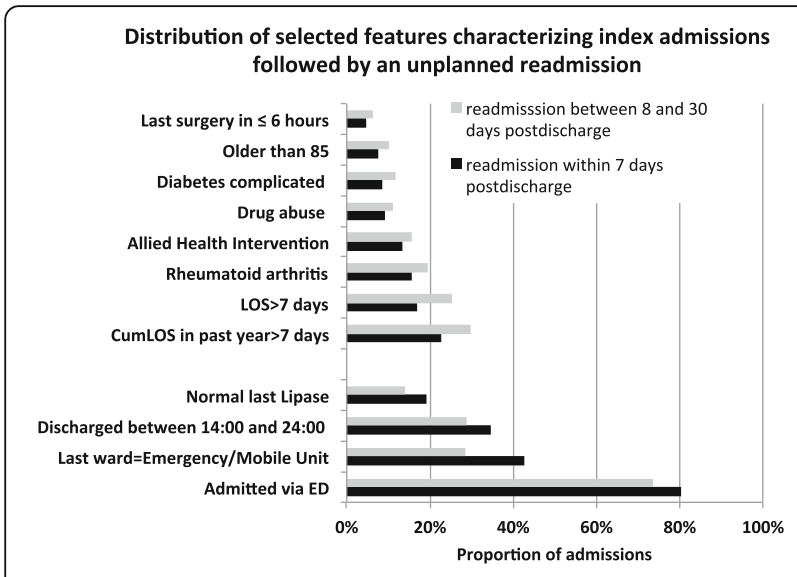

Fig. 3 Distribution of selected features characterizing index admissions that are followed by unplanned readmission within 7 days from discharge or unplanned readmission between 8 and 30 days from discharge. Selected features are those for which the difference in proportions is statistically significant. LOS = Length of Stay; CumLOS=Cummulative LOS; $\mathrm{ED}=$ Emergency Department; Emergency/Mobile ward refers to Emergency ward or mobile acute treatment units

available published models. Our choice of threshold to stratify patients into two groups (no unplanned readmission and unplanned readmission) was built to optimize the combined sum of sensitivity or recall and specificity. An alternative threshold could have been chosen to optimize PPV. For example, a higher cut-off score of 20 points in RETURN30 (see Additional file 1: Table S8) provided $\mathrm{PPV}=28 \%$ but a Sensitivity $=18 \%$. Other scores like e.g. PARR-30 [24] reported a PPV $=59 \%$ and Sensitivity $=5.4 \%$ for scores above $50 \%$. A high sensitivity ensures that most patients at high risk of readmission are correctly identified. On the other hand, a high PPV could contain the costs of readmission strategies since it ensures patients selected for an intervention are likely to benefit from it.

In this study we make use of a modelling technique known as gradient tree boosting [29]; uncommon in the construction of previous readmission models, but popular in the machine learning community. Although performing variable selection with this algorithm does not appear to provide improved predictive performance when compared to previous work, existing high-performing models were trained in much larger datasets. Further work is needed to assess if performance here could improve with larger sample sizes.

In addition, due to the fact that outcome categories are very unequally represented, with a much larger number of no readmissions than unplanned readmissions, it was easier to predict unplanned readmissions to hospital within 60 days post-discharge $(\mathrm{AUC}=0.74)$ than within 7 days $(\mathrm{AUC}=0.71)$.
A patient's history of health care utilization in the previous year was found to be the most important predictor of unplanned readmission in all models. This agrees with the previous literature which found number of hospital admissions [21, 23-25], number of emergency department visits [20,21], and number of primary care and specialist visits [25] in the past year to be important predictors. Number of previous hospital admissions was strongly correlated with cumulative LOS across these admissions. The latter was chosen as the better proxy for acute care utilization. Similarly, the number of days since last admission was a common predictor found in previous work [24, 25], as was urgency of the index admission [20, 21, 23]. Unmarried patients and public (Medicare-holder) patients were more likely to have an unplanned readmission. Australia's publicly funded health care system entitles citizens and most permanent residents to be eligible for Medicare. Medicare services include treatment in public hospitals, subsidised treatment in private hospitals, subsidised outpatient services and subsidised access to medicines prescribed in private hospitals and the community.

Abnormal results for commonly performed pathology tests (in particular within the FBC, UEC and LFT panels) before discharge were also found to be important features. This agrees with Donze et al. [23], who found low haemoglobin and low sodium at discharge to be predictive of potentially avoidable readmissions. Another similarity with Donze et al., is the identification of a cancer diagnosis as a predictor of unplanned readmission. Several previous studies have found high rates of unplanned readmissions for cancer patients [36-38]. Analysis of risk factors for these patients pointed at severity of illness and procedure complications as reasons for these high rates. These findings raise the issue of improving oncology care in primary and community care. Potentially preventable hospitalizations for very ill cancer patients and the need to improve access to palliative care outside hospitals has also been discussed in the context of the 'weekend effect' [39] (difference in mortality observed in patients admitted to hospital during the weekend versus weekdays).

We found that some predictors of readmission within 30 and 60 days post-discharge (such as LOS and allied health intervention) were not relevant for shorter-term predictions. Conversely, time since last surgery, last ward and discharge time were predictors of unplanned readmission within 7 days post-discharge but did not affect the prediction of longer-term readmissions. Analysis of predictors' distributions in the group that had a 7-day readmission versus 8 to 30 -day readmission confirmed that longer-term readmissions where more frequently associated with older patients, longer hospital 
stays, higher use of acute care in the past year and more comorbidities; while short-term readmissions were more frequently associated with urgent admissions. This is an indication that some shorter-term readmissions may have different causality than longer-term readmissions.

\section{Limitations and future work}

In this study, a readmission is considered 'unplanned' if it takes place via the Emergency Department. By using this definition, we may be missing unplanned admissions to hospital initiated by specialists, who directly admit their patients into hospital prompted by unexpected events. Furthermore, an unplanned readmission does not necessarily imply preventability. A modification of our models considering validated definitions of potentially preventable readmission is left as future work. Although several definitions of potentially preventable readmissions have been put forward [40, 41], current readmission models have not been compared using the same definition. Standardising this concept would lead to appropriate comparisons across predictive techniques and their corresponding scores. More importantly, current scores only have fair discrimination ability. Inclusion of more informative data elements should be taken into account if we are to use these scores in clinical practice.

The robustness of the risk scores, particularly regarding the weights of the less important predictors can be improved with larger training datasets. This is particularly the case in the 7-day readmission model, where less than 2000 unplanned readmissions are available in the derivation set. Also, the effect of discretising continuous variables was not explored in this study and may have influenced the prediction performance of the scores. This study did not include any investigation to establish causality between predictors and unplanned readmission. Furthermore, this study is limited to index admissions to a 350-bed teaching, metropolitan hospital in Sydney. Therefore, population characteristics reflect those of the catchment area of this hospital. The inclusion of larger sample sizes, investigation of causality for selected predictors and external validation using records from different hospitals can produce more robust and clinically meaningful scores. This has been left for future work.

\section{Conclusions}

This study developed risk scores to identify 7-day, 30day and 60-day all-cause unplanned readmission in a Sydney hospital. The models achieved a fair predictive performance, similar to current models trained with larger datasets. Additional variables not currently contained in EHR data may be needed to improve performance. There is some indication that 7-day unplanned readmissions may have different causal pathways than longer- term readmissions. Overall, it may be more beneficial to design screening tools that identify candidates for appropriate preventive interventions, such as candidates that may benefit from delayed discharge, or candidates that should be offered alternative care pathways.

\section{Additional file}

\begin{abstract}
Additional file 1: Table S1. Parameters of the Gradient Tree Boosting algorithm. In this study, we used the freely available gradient tree boosting algorithm implemented in the R package XGBoost with the following parameters chosen via manual tuning. Table S2. Conversion of continuous variables into categorical variables: cutting points for hospital length of stay (LOS), age (years), cumulative LOS (hours) in the previous year, days from last admission, number of pathology tests, number of pathology panels, hours since last surgery, hours since last panel and admission type. Table S3: Characteristics of patients and their hospital admissions for the study population. Main descriptive statistics. Table S4. Main categories of primary diagnosis (ICD10-AM) in our cohort. Table S5. Comorbidity groups in our cohort (Reference value $=$ no comorbidity). Table S6. Pathology variables identified by the hospital laboratory in our cohort (Reference value $=$ missing). Table S7. Calibration performance; Observed and expected rates for selected scores can be found in this table. Table S8. Sensitivity, specificity and PPV for different cut-off scores. (PDF 155 kb)
\end{abstract}

\begin{abstract}
Abbreviations
APDC: Admitted Patient Data Collection; AUC: Area under the receiver operating characteristic curve; CRP: C-Reactive Protein; CumLOS: Cumulative length of stay in the last year from index admission; ED: Emergency Department; EDDC: Emergency Department Data Collection; EHR: Electronic Health Record; FBC: Full blood count; HOSPITAL: "H" stands for hemoglobin at discharge; "O" discharge from an oncology service; "S" sodium level at discharge; "P" procedure during the index admission; "I" index "T" type of admission; " $A$ " number of admissions during the last 12 months; " $L$ " length of stay; INR: International normalized ratio; LACE: "L" stands for length of stay of the index admission; " $A$ ": acuity of the admission; " $C$ ": comorbidity; " $E$ ": Emergency Department visits in the last 6 months; LFT: Liver Function Tests; LOS: Length of stay; NSW: New South Wales; PARR - 30: Patients at risk of readmission within 30 days; PPV: Positive predictive value; PREADM: Preadmission readmission detection model; RBDM: Registry of Births, Deaths and Marriages; UEC: Urea, Electrolytes and Creatinine; XGBoost: eXtrem Gradient Boosting
\end{abstract}

\section{Acknowledgments}

We would like to acknowledge the Australian National Health and Medical Research Council (NHMRC) and the St Vincent's Clinic Foundation for the funding that made this study possible. For their help in the provision and linkage of the data, we would like to acknowledge the New South Wales Ministry of Health, the hospital IT personnel and the New South Wales Centre for Record Linkage.

YM, OPC and BG elaborated this paper with funds provided by the Australian National Health and Medical Research Council (NHMRC) and the St Vincent's Clinic Foundation. The funding bodies did not have any role in any aspect of this research or preparation of the manuscript for publication.

\section{Funding}

This work was funded by the Australian National Health and Medical Research Council (NHMRC) and the St Vincent's Clinic Foundation. Its contents are the responsibility of the authors and do not reflect the views of the research granting bodies. Data used in this study was provided by the New South Wales Ministry of Health, and the IT hospital personnel, and data linkage was performed by the New South Wales Centre for Record Linkage. The authors were responsible for the data analysis after the extraction and linkage. 


\section{Availability of data and materials}

The NSW Admitted Patient Data Collection (APDC), the NSW Emergency Department Data Collection (EDDC), and the NSW Registry of Births, Deaths and Marriages (RBDM) and their respective linkage is available from the NSW Centre for Record Linkage (http://www.cherel.org.au). The Electronic Health Records from 77,776 patients admitted to a 350-bed Sydney teaching were used under license for the current study and considered protected health data under Australian laws, and so are not publicly available.

\section{Authors' contributions}

Contributors YM and BG: contributed to conception and design, analysis and interpretation of the data and results, drafting and writing the article, revising it critically for important intellectual content and final approval of the version to be published. OPC contributed to conception of the paper, preparation of the data, preliminary analysis of the data as well as revising the paper critically for important intellectual and content. DR and RD: contributed to interpretation of the data and revising the paper critically for important intellectual and content. EC: contributed to supervision of the paper, developed hypotheses, analysis and interpretation of the data and results, as well as drafting and writing the article, revising it critically for important intellectual content and final approval of the version to be published. All authors read and approved the final manuscript.

\section{Ethics approval and consent to participate}

Ethics approval was obtained from New South Wales Population and Health Services Research Ethics Committee and the hospital's Ethics Committee (HREC/13/CIPHS/29). The need for consent from the participants was waived by the ethics committee due to the retrospective nature of the analysis.

\section{Consent for publication}

Not applicable.

\section{Competing interests}

The authors declare that they have no competing interests.

\section{Publisher's Note}

Springer Nature remains neutral with regard to jurisdictional claims in published maps and institutional affiliations.

\section{Author details}

${ }^{1}$ Centre for Health Informatics, Australian Institute of Health Innovation, Macquarie University, Level 6, 75 Talavera Rd, Sydney, NSW 2109, Australia. ${ }^{2}$ Centre for Big Data Research in Health, University of New South Wales, Level 1, AGSM Building (G27), Sydney, NSW 2052, Australia. ${ }^{3}$ Chief Information Officer, St Vincent's Health Australia, Sydney, NSW 2010, Australia. ${ }^{4}$ Clinical Pharmacology and Toxicology, St Vincent's Hospital, Sydney, NSW 2010, Australia. ${ }^{5}$ St Vincent's Clinical School, St Vincent's Hospital, University of New South Wales, Sydney, Australia.

Received: 25 March 2017 Accepted: 29 December 2017

Published online: 04 January 2018

\section{References}

1. Zhou H, Della PR, Roberts P, Goh L, Dhaliwal SS. Utility of models to predict 28-day or 30-day unplanned hospital readmissions: an updated systematic review. BMJ Open. 2016;6(6)::011060.

2. Joynt KE, Jha AK. A path forward on Medicare readmissions. N Engl J Med. 2013;368(13):1175-7.

3. Refining the hospital readmissions reduction program. Report to the Congress: Medicare and the Health Care Delivery System. ch. 2013;4:91-114. Medicare Payment Advisory Commission. Washington, D.C., United States of America. http://www.medpac.gov/docs/default-source/reports/jun13_ch04.pdf.

4. Spotlight on measurement: return to acute care following hospitalisation. Spotlight on readmissions. Sydney, NSW: BHI. July 2009 - June 2012.

5. Joynt KE, Jha AK. Thirty-day readmissions - truth and consequences. N Engl J Med. 2012;366(15):1366-9.

6. Blunt I, Bardsley M, Grove A, Clarke, A. Classifying emergency 30-day readmissions in England using routine hospital data 2004-2010: what is the scope for reduction?. Emerg Med J. 2015;32(1):44-50. Published online 2014 Mar 25. doi:https://doi.org/10.1136/emermed-2013-202531.
7. Canadian Institute for Health Information, All-Cause Readmission to Acute Care and Return to the Emergency Department (Ottawa, Ont:: CIHI, 2012).

8. Silverstein MD, Qin H, Mercer SQ, Fong J, Haydar Z, editors. Risk factors for 30-day hospital readmission in patients? 65 years of age. Baylor University Medical Center Proceedings; 2008: Baylor University Medical Center.

9. Kansagara D, Englander H, Salanitro A, Kagen D, Theobald C, Freeman M, et al. Risk prediction models for hospital readmission: a systematic review. JAMA. 2011;306(15):1688-98.

10. García-Pérez L, Linertová R, Lorenzo-Riera A, Vázquez-Díaz J, Duque-González B, Sarría-Santamera A. Risk factors for hospital readmissions in elderly patients: a systematic review. QJM. 2011;104(8):639-51.

11. Nolte E, Roland MO, Guthrie S, Brereton L, Europe R. Preventing emergency readmissions to hospital: a scoping review. 2010.

12. Hoyer EH, Needham DM, Miller J, Deutschendorf A, Friedman M, Brotman DJ. Functional status impairment is associated with unplanned readmissions. Arch Phys Med Rehabil. 2013;94(10):1951-8.

13. Shulan M, Gao K, Moore CD. Predicting 30-day all-cause hospital readmissions. Health Care Manag Sci. 2013;16(2):167-75.

14. Hu J, Gonsahn MD, Nerenz DR. Socioeconomic status and readmissions: evidence from an urban teaching hospital. Health Aff. 2014;33(5):778-85.

15. Glance LG, Kellermann AL, Osler TM, Li Y, Mukamel DB, Lustik SJ, et al. Hospital readmission after noncardiac surgery: the role of major complications. JAMA Surg. 2014;149(5):439-45.

16. Leppin AL, Gionfriddo MR, Kessler M, Brito JP, Mair FS, Gallacher K, et al. Preventing 30-day hospital readmissions: a systematic review and metaanalysis of randomized trials. JAMA Intern Med. 2014;174(7):1095-107.

17. Bradley EH, Sipsma H, Horwitz LI, Curry L, Krumholz HM. Contemporary data about hospital strategies to reduce unplanned readmissions: what has changed? JAMA Intern Med. 2014;174(1):154-6.

18. van Walraven C, Jennings A, Taljaard M, Dhalla I, English S, Mulpuru S, et al. Incidence of potentially avoidable urgent readmissions and their relation to all-cause urgent readmissions. Can Med Assoc J. 2011;183(14):E1067-E72.

19. Healthwatch. Healthwatch England Special Inquiry: Safely home. http:// www.healthwatch.co.uk. 2015.

20. van Walraven C, Dhalla IA, Bell C, Etchells E, Stiell IG, Zarnke K, et al. Derivation and validation of an index to predict early death or unplanned readmission after discharge from hospital to the community. Can Med Assoc J. 2010;182(6):551-7.

21. van Walraven C, Wong J, Forster A. LACE+ index: extension of a validated index to predict early death or urgent readmission after hospital discharge using administrative data. Open Med. 2012;6(3):80-9.

22. Bradley E, Yakusheva O, Horwitz LI, Sipsma H, Fletcher J. Identifying patients at increased risk for unplanned readmission. Med Care. 2013;51(9):761.

23. Donzé J, Aujesky D, Williams D, Schnipper JL. Potentially avoidable 30-day hospital readmissions in medical patients: derivation and validation of a prediction model. JAMA Intern Med. 2013;173(8):632-8.

24. Billings J, Blunt I, Steventon A, Georghiou T, Lewis G, Bardsley M. Development of a predictive model to identify inpatients at risk of re-admission within 30 days of discharge (PARR-30). BMJ Open. 2012;2(4):e001667.

25. Shadmi E, Flaks-Manov N, Hoshen M, Goldman O, Bitterman H, Balicer RD, et al. Predicting 30-day readmissions with preadmission electronic health record data. Med Care. 2015;53(3):283-9.

26. Cai X, Perez-Concha O, Coiera E, Martin-Sanchez F, Day R, Roffe D, Gallego B. Real-time prediction of mortality, readmission, and length of stay using electronic health record data. Journal of the American Medical Informatics Association. 2015;23(3):553-61.

27. Tabak YP, Sun X, Nunez CM, Johannes RS. Using electronic health record data to develop inpatient mortality predictive model: acute laboratory risk of mortality score (ALaRMS). J Am Med Inform Assoc. 2014;21(3):455-63.

28. Nguyen OK, Halm EA, Makam AN. Further limitations of the HOSPITAL score in US hospitals. JAMA Intern Med. 2016;176(8):1232-3.

29. Friedman JH. Greedy function approximation: a gradient boosting machine. Annals of statistics. 2015;29(5):1189-1232.

30. Caruana R, Niculescu-Mizil A. An empirical comparison of supervised learning algorithms. Proceeding ICML '06 Proceedings of the 23rd international conference on Machine learning. Pages 161-168 2006.

31. Lawrence G, Dinh I, Taylor L. The Centre for Health Record Linkage: a new resource for health services research and evaluation. Health Info Manag J. 2008;37(2):60,

32. Elixhauser A, Steiner C, Harris DR, Coffey RM. Comorbidity measures for use with administrative data. Med Care. 1998;36(1):8-27. 
33. Chen T, He T. xgboost: eXtreme Gradient Boosting. R package version 04-2. 2015.

34. Gruneir A, Dhalla IA, van Walraven C, Fischer HD, Camacho X, Rochon P. Unplanned readmissions after hospital discharge among patients identified as being at high risk for readmission using a validated predictive algorithm. Open Med. 2011;5(2):104-11.

35. Swain MJ, Kharrazi H. Feasibility of 30-day hospital readmission prediction modeling based on health information exchange data. Int J Med Inform. 2015;84(12):1048-56

36. Saunders ND, Nichols SD, Antiporda MA, Johnson K, Walker K, Nilsson R, et al. Examination of unplanned 30-day readmissions to a comprehensive cancer hospital. J Oncol Pract. 2015;11(2):e177-e81.

37. Manzano J-GM, Gadiraju S, Hiremath A, Lin HY, Farroni J, Halm J. Unplanned 30-day readmissions in a general internal medicine hospitalist Service at a Comprehensive Cancer Center. J Oncol Pract. 2015;11(5):410-5.

38. Ji H, Abushomar H, Chen X, Qian C, Gerson D. All-cause readmission to acute care for cancer patients. Healthc Q (Toronto, Ont). 2011;15(3):14-6.

39. Perez-Concha O, Gallego B, Hillman K, Delaney GP, Coiera E. Do variations in hospital mortality patterns after weekend admission reflect reduced quality of care or different patient cohorts? A population-based study. BMJ Qual Saf. 2014;23(3):215-22.

40. Halfon P, Eggli Y, Prêtre-Rohrbach I, Meylan D, Marazzi A, Burnand B. Validation of the potentially avoidable hospital readmission rate as a routine indicator of the quality of hospital care. Med Care. 2006:44(11):972-81.

41. Halfon P, Eggli Y, van Melle G, Chevalier J, Wasserfallen J-B, Burnand B. Measuring potentially avoidable hospital readmissions. J Clin Epidemiol. 2002;55(6):573-87.

\section{Submit your next manuscript to BioMed Central and we will help you at every step:}

- We accept pre-submission inquiries

- Our selector tool helps you to find the most relevant journal

- We provide round the clock customer support

- Convenient online submission

- Thorough peer review

- Inclusion in PubMed and all major indexing services

- Maximum visibility for your research

Submit your manuscript at www.biomedcentral.com/submit 